\title{
AN INTERDISCIPLINARY APPROACH FOR THE SEISMIC VULNERABILITY ASSESSMENT OF HISTORICAL CENTRES IN MASONRY BUILDING AGGREGATES: APPLICATION TO THE CITY OF SCARPERIA, ITALY
}

\author{
V. Cardinali ${ }^{1}$, M.T. Cristofaro ${ }^{1}$, M. Ferrini ${ }^{1}$, R. Nudo ${ }^{1}$, B. Paoletti ${ }^{1}$, M. Tanganelli ${ }^{1, *}$ \\ ${ }^{1}$ Dipartimento di Architettura, Università degli studi di Firenze - (vieri.cardinali, mariateresa.cristofaro, maurizio.ferrini, \\ raffaele.nudo, barbara.paoletti, marco.tanganelli)@unifi.it
}

Commission II - WG II/8

KEYWORDS: Seismic vulnerability, Masonry building aggregates, Historical centres, Urban Scale, Multidisciplinary approach

\begin{abstract}
:
The seismic vulnerability of masonry building aggregates is very difficult to determine, since it is affected by many uncertainties. The most uncertain quantities concern the historical periodization of structural aggregates. Moreover, the studies made at the urban scale can hardly be thorough, and usually the knowledge achieved on the single units is not fully satisfactory, so that the structural designer has to deal with uncompleted architectonical surveys and partial data; one of the most important problems concerns the lack of knowledge about the boundary conditions between adjacent structures. In order to perform mechanical analyses, an extensive knowledge of materials and techniques adopted is required. In this paper, an integrated methodology for the seismic assessment of building aggregate is presented. It concerns a multidisciplinary knowledge-based approach calibrated over the historical centres and the urban aggregates; the procedure joins different aspects, such as the use of modern technologies for an integrated knowledge, plans reconstructions through archival documents, laser scanner digital survey of urban fronts, non-destructive investigations of the materials. GIS and BIM platforms have been used to implement and collect data in order to perform detailed analyses. The information allowed to assess the seismic vulnerability of the building aggregates and the expected damage scenarios through empirical methodologies. The city of Scarperia, founded a few kilometres from Florence during the Medieval Age and characterized by a medium seismicity, has been chosen as a case study for the presented procedure.
\end{abstract}

\section{INTRODUCTION}

\subsection{Issues concerning the state of art}

In the last decades, the vulnerability assessment of existing urban stocks has been extensively studied. Nowadays, the seismic performance of a single independent masonry building can be studied by well-defined methodologies, recognized in codes as "semi-probabilistic approaches" (FEMA, EC8); nevertheless, the assessment of masonry buildings at the urban scale passes through a higher level of uncertainties. In this area of interest, masonry building aggregates still represent a developable topic. Due to the interactions between structural components, which depend, in turn, on the relevance of their connections, this theme still represents an open research field. During the last years many studies have been done in order to assess the seismic performance of masonry buildings (Galassi et al., 2018; D'Altri et al., 2019). In terms of modelling, they concerned different scales of discretization of masonry walls, based on different approaches. Changing the scale of interest from the building to the aggregate, the complexity of the problems increases. Uncertainties related to the masonry quality and their intrinsic mechanical characteristics grow, the lack of knowledge in terms of geometrical and structural features becomes relevant; finally, computational effort of sophisticated nonlinear analyses assume unsustainable amounts. For this reason, the scientific community has developed hybrid procedures in order to limit the mechanical analysis to specific and selected case-studies, aiming to extend the results to broader families of buildings because of their similarities.
Based on the above considerations, the proposed work aims to develop an interdisciplinary methodological approach in order to assess the seismic vulnerability of building aggregates. The presented procedure regards two different levels of evaluation, considering the city scale and the aggregate scale. These deepening studies are correlated by in-depth management systems in order to carry out different vulnerability analyses. Starting from the definition of macro-seismic classification and the acquisition in a Geographical Information System (GIS) involving different data sources, the change of scale passes through the management of the aggregate into a Building Information Modelling (BIM) and the performance of more refined analyses. The final step (not presented in this work for sake of brevity) would concern the building itself and involves parametric and mechanical analyses; each step establishes the implementation of the results obtained at larger scales and their interpretation. The final outcomes of the analyses concern the assessment of the seismic performance of the building aggregates, the definition of vulnerability curves and the related damage scenarios. The presented procedure has been applied to the city centre of Scarperia, in Tuscany. The city, built over a regular layout starting from the 14th century, is characterized by a medium-high seismic hazard and has been hit by important earthquakes over the centuries. The last important ground motion, having a magnitude of about 6.0, occurred in June 1919 and made severe damages to the buildings; on 9 December 2019, a century later, the Mugello area has been hit by an earthquake of about 4.5 intensity, with limited damages to constructions. During this time, the urban layout has undergone some alterations that need to be investigated.

\footnotetext{
* Corresponding author
} 


\subsection{Current methodologies for the seismic vulnerability at urban scale}

Safety of urban areas is one of the most important challenges of the scientific community during the last decades. Seismic assessment can be referred to individual structures or evaluated at the urban scale. In the second case, which is the object of this project, the seismic vulnerability is defined as a weakness, or incapacity to provide an adequate response, or a possible lack of resilience in components of the city when exposed to seismic motions over a given period of time (Martínez-Cuevas et al., 2017). An urban scale approach cannot expect to evaluate the overall urban stock as it is possible to do for a single building. Its use, based on statistical considerations, needs to avoid large-scale computational efforts with sophisticated individual analyses. According to the literature, methodologies at urban scale can be divided into three different classes: empirical, analytical and hybrid methodologies. Empirical methodologies are the basic ones. Different procedures have been defined in last years, starting from the observation of damage produced by past earthquakes in buildings. EMS-98 (Grunthal, 1998) and the GNDT (Gruppo Nazionale Difesa dai Terremoti) researches, developed in 80's both for masonry and RC buildings, represent important contributions. Analytical techniques are mostly used for single buildings and concern mechanical models to perform seismic analyses. Finally, hybrid approaches identify representative buildings to perform analytical analyses in order to extend their results to a broader class of homogeneous buildings. The meaningfulness of the obtained results is related to the quality of the performed buildings classification, which should be detailed enough to account for the specificities of the considered buildings but general enough to cover a significant number of cases. Vicente et al. (2011) have defined the approaches as direct, indirect, conventional and hybrid techniques. Seismic vulnerability of structures at urban scale can be evaluated using qualitative descriptors of specific macro-seismic scales, vulnerability indexes and capacity curves. The expected physical damage can be expressed by damage probability matrices, vulnerability functions and fragility curves (Lantada et al., 2009; Maio et al., 2015; Bento 2019).

The management of urban areas is a target for municipalities and institutions to control urban stocks in order to deal with emergency and risk scenarios. In this context GIS is the most used; through the superimposition of several layers lead to different aspects it is possible to manage the complexity of urban areas suggesting integrated visions. In the last years many studies have been conducted allowing to define several proposals (Falção et al., 2018; Cavaleri et al., 2017).

\subsection{Further developments for the use of empirical methodologies at urban scale}

If GIS software showed the possibility of data management at urban scale, concerning the individual buildings, and especially referred to the Cultural Heritage Buildings, the Historical BIM (H-BIM) is actually the most used methodology (Talon et al., 2017). Compared to GIS, it allows a higher Level of Development/Detail (LOD) that distinguishes its operability for intervention and restoration projects. By the way, the use of such informative 3-D models is not strictly related to the maintenance of the buildings, allowing, for example, information facilities for museal paths or similar. Nowadays, some examples related to the seismic vulnerability of existing buildings through empirical procedures implemented in BIM are presented in literature (Colucci et al., 2018), as procedures including the use of BIM in GIS environments (Matrone et al., 2019). The most-likely future for the empirical methodologies will be related to the enhancement of the level of accuracy of such types of analyses, connected with more refined data acquisitions.

\subsection{Analysis of masonry building aggregates}

Assessment of historical building aggregates is one of the most contemporary themes in the seismic engineering. Nowadays indeed, the scientific community has become aware of the complexity evaluating the seismic response of structural aggregates (Greco et al., 2018; Senaldi et al., 2010, 2019; Ruggieri et al., 2018; Valente et al., 2019). It is worth noting that structural interactions are strictly related to the quality of connections among structural components, such as walls and floors. Walls grown side by side can provide hammering phenomena between the architectonical cells; slabs (with different values of stiffness), when not located at the same levels, can occur damages in the side walls and determine collapse of certain zones of the aggregate. Nevertheless, the regularity in height of the buildings can help the in-plane response of the aggregates but, at the same time, corner structures always suffer more the influence of the adjacent constructions. In any case, in buildings aggregates, attention to connections between walls in order to prevent local damage and out-of-plane mechanisms and, in general, accuracy in the workmanship, are always valid.

\section{SCARPERIA}

\subsection{Historical genesis}

The city of Scarperia, located in the Mugello area, was founded in the 1306 by the Florentine Republic for the control of the viability between Florence and northern Italy. Its urban asset is typical of the so called "città nuove" of the period: a linear scheme based on two main streets defining a longitudinal axis in plan and a transversal one. The intersection between the two roads generated the main square of the city, dominated by the Palazzo de' Vicari, a historical building representative of the local political power. In Figure 1 the main characteristics of the urban layout of the historical city are showed.

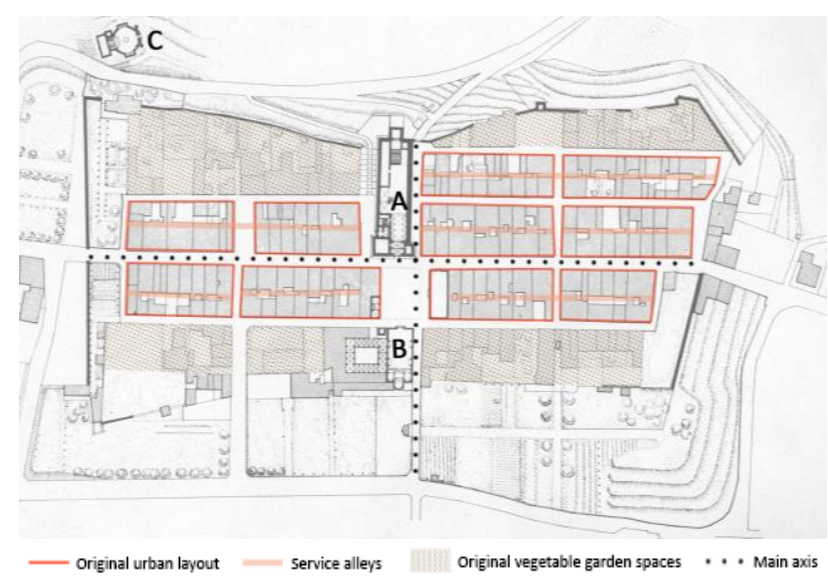

Figure 1. Original urban layout of Scarperia.

A) Palazzo dei Vicari;

B) Vecchia Propositura;

C) Oratorio della Madonna del Vivaio. 


\subsection{Seismogenetic history}

The Mugello area is characterized by a medium-high seismic risk. This is due to the presence of several active faults that activated remarkable earthquakes during the ages. The most important seismic events that hit Scarperia are dated 13/09/1542 and 29/06/1919; they have been classified as IX and VII/VIII of the MCS scale, with magnitudes around the grade 6 of the Richter Scale.

The 1542 event, reported in the historical chronicles, is considered the most important earthquake that shook the area. Many important and well-done palaces together with the ordinary buildings collapsed. Hundreds of people dead. The earthquake of 1919 had his epicenter in Vicchio (close to Scarperia) and represents one of the most important seismic events of the Appenino Tosco-Romagnolo and the Mugello area (Magnitude $=6.2$ ). In the waste area, more than one hundred victims was registered, with over four-hundreds injured people. The houses collapsed or affected by heavy damage were over eight hundred. In the Scarperia municipality numerous buildings suffered severe damages; in the historical walls, in the Palazzo de' Vicari and many churches partial failures occurred.

\section{KNOWLEDGE-BASED APPROACH}

The presented procedure aims to combine different scale of interest in order to define the seismic vulnerability assessment of urban aggregates. To this purpose, three different scales have been defined. Starting from an "urban scale" approach (US), the knowledge-based studies present in-depth analyses reducing the scale of interest. At the US scale a GIS platform and the macroseismic approach has been used. At the "aggregate level" (AL), a structural aggregate has been selected as representative of the stock of Scarperia and more in-depth studies have been conducted. A BIM database has been finally used in order to gather the information collected. A laser scanner survey integrated by direct in-situ inspections and cadastral reconstructions of the internal parts of the aggregate allowed performing a parametric modelling. At the AL, the GNDT second level assessment procedure implemented by Formisano et al. (2011) has been adopted. The BIM platform allows both the existence of a 3-D database for the management of the existing heritage (H-BIM), and the possibility to export geometrical and mechanical information obtained in order to perform mechanical analyses. These phases are at the basis of any further intervention for strengthening and retrofitting of the buildings, where, in H-BIM optic, the database should be still upgraded.

\subsection{Urban Scale approach}

The following procedure has involved different systems of management to collect different sources of data. Starting from the urban scale, Geographic Information System (GIS) has been used. The Technical Regional Map (TRM) has been collected through the OpenSource Software QGis. GIS software are always more used in the field of the seismic risk and resilience of urban areas; in fact, they allow to manage different source of data combining themselves to offer new interpretations and views (Catulo et al., 2018). The facility of use combined to the creation of efficient files able to support a relevant number of information is perfectly coherent with the use for the assessment of the seismic vulnerability of the buildings. The work gathered the collection data into the categories presented in the EMS-98 (Grunthal 1998) scale assuming the modifiers introduced by Giovinazzi and
Lagomarsino (2004). The first operations consisted in the cleaning of the shapefiles provided by TRM in order to represent each structural aggregate with closed polygons. Finally, several information has been assigned to each shapefile.

3.1.1 The data acquisition: This operation regarded numerous phases made both through digital acquisition and consulting, both performed in-situ in the centre of Scarperia. The first part concerns the acquisition of the historical evolution of the city and of the main drawings and documents of Scarperia. Then, a cadastral reconstruction of each buildings and each apartment has been made considering the entire number of floors presented in the city centre.

Both photogrammetric and direct surveys have been made for the external parts of the city and for the façades of buildings. The information collected through in-situ campaigns, starting with the cadastral data, have been implemented and a detailed survey of the entire city centre has been made. It is well-known that one of the most difficult phases of US aims is the obtainment of geometrical and mechanical information regarding the buildings. In fact, the various properties that composes our cities represent in this case a hindrance to the possibility to get complete surveys so that the lack of knowledge becomes particularly relevant.

This phase has been correlated with the use of non-destructive techniques in order to implement the data obtained from the historical researches and the surveys. A relevant thermography campaign has been performed. It concerned all the external walls of the buildings in order to check the wall composition and the presence of irregularities. The investigation did not provide always the same results. In fact, thermography campaigns are sensitive to the object investigated (depth of the plaster mortar, presence of wiring or other systems that focus the camera over wrong ranges of colours) and to the boundary conditions (such as walls enlightened by the Sun or not, the hour of the performance etc.).

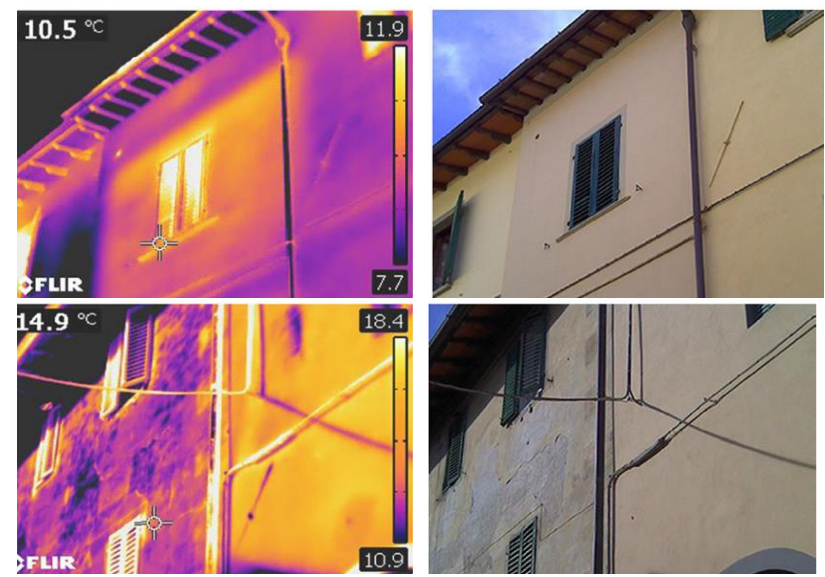

Figure 2. Thermography campaign; it has been made using a camera Flir T4xx.

By the way, in some case the thermography campaign can help to better define the materials composing the bearing walls and their disposition (irregular rubble stone, presence of horizontal courses, regular clay brick walls), catching even details of ancient openings hidden by the plaster surfaces. Figure 2 shows some example of the Thermography campaign made all around the Historical centre of Scarperia. 
3.1.2 Macroscale assessment: The vulnerability assessment of the centre of Scarperia has been expressed through a macroseismic scale. As already said, the work has been implemented in a GIS software using the modifiers proposed by Giovinazzi and Lagomarsino (2004). This passed through an interdisciplinary approach involving historical researches, digital surveys, non-destructive techniques and in-situ investigations. The application of the method provides a Vulnerability Index V; finally, using the formula (1) presented in literature, a Vulnerability curve can be defined to express the expected level of damage for each level of seismic intensity I.

$\mu_{D}=2.5\left[1+\tanh \left(\frac{I+6.25 V-13.1}{Q}\right)\right] ; 0 \leq \mu_{D} \leq 5$

$\mu_{D}$ is the expected level of damage expressed in macroseismic scale and ranging between 0 (No Damage) to 5 (Collapse); the vulnerability curve is then expressed through the hyperbolic tangent of a ratio that combine the Intensity level of the expected earthquake (ranging between 0 and 12), the Vulnerability Index already defined and a Ductility Factor Q. Different values for Q can be assumed; in this work, a $\mathrm{Q}$ value equal to 2.3 following the Lagomarsino suggestions has been adopted (2006). In Figure 3 the results of vulnerability curves for the city of Scarperia are reported. In the graphic the mean value obtained from the 18 aggregates are plotted, while in Table 1 the mean results with their standard deviations are shown.

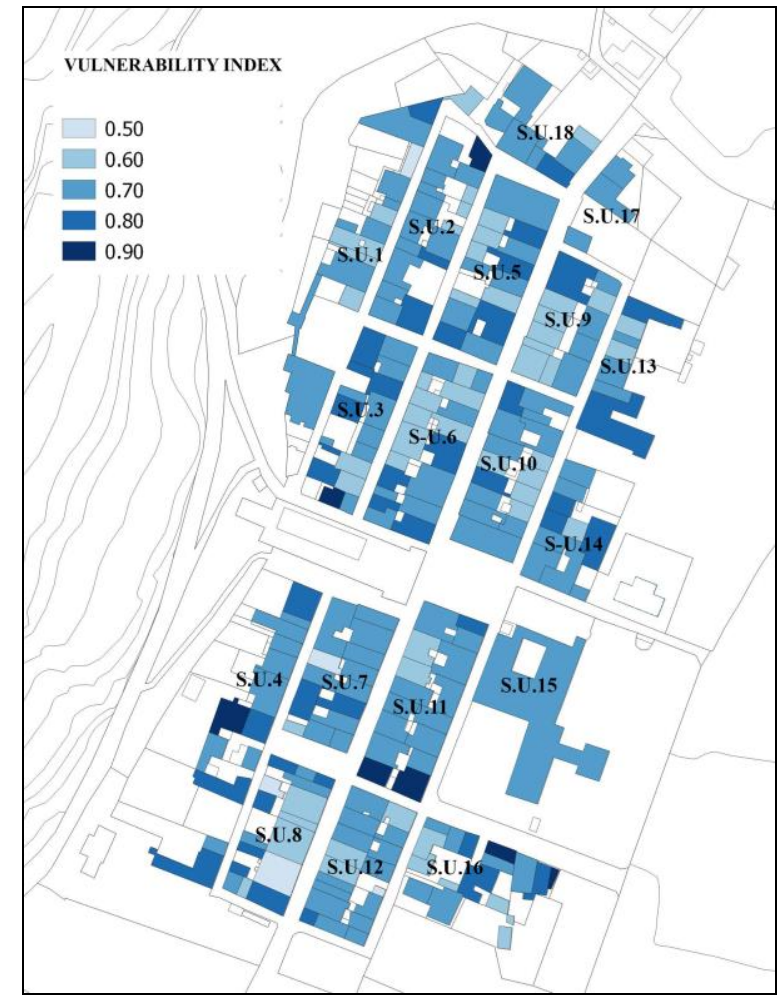

Figure 3. Macroseismic Vulnerability Index of a part of the city of Scarperia. The results are extracted from the GIS platform.

Scarperia is a city characterized by a medium-high seismicity. From a macroseismic point of view, it can be considered ranging between level VII and IX. The mean vulnerability curve is generated by a V index equal to 0.72 with a standard deviation of 0.108 . The relative typological vulnerability curve is plotted in Figure 4.
Finally, the probability that a certain damage occurs for a given level of seismic intensity I has been expressed according the probability mass function of the binomial distribution:

PMF : $p k=\frac{n !}{k !(n-k) !} \cdot d_{k} \cdot(1-d)^{n-k} n \geq 0$

Results are finally expressed in Figure 5 considering the mean value of $\mathrm{V}$ and the binomial distribution for different levels of seismic intensity (from grade VI to XII).

\begin{tabular}{|l|l|l|}
\hline S.U. & V mean & St. dev. \\
\hline 1 & 0.67 & 0.738 \\
2 & 0.69 & 0.07 \\
3 & 0.73 & 0.0717 \\
4 & 0.79 & 0.068 \\
5 & 0.70 & 0.085 \\
6 & 0.69 & 0.081 \\
7 & 0.72 & 0.070 \\
8 & 0.73 & 0.093 \\
9 & 0.66 & 0.0712 \\
10 & 0.69 & 0.069 \\
11 & 0.76 & 0.085 \\
12 & 0.73 & 0.06 \\
13 & 0.74 & 0.065 \\
14 & 0.77 & 0.058 \\
15 & - & - \\
16 & 0.74 & 0.085 \\
17 & 0.74 & 0 \\
18 & 0.69 & 0.0738 \\
\hline
\end{tabular}

Table 1. V index for the different aggregates of Scarperia.

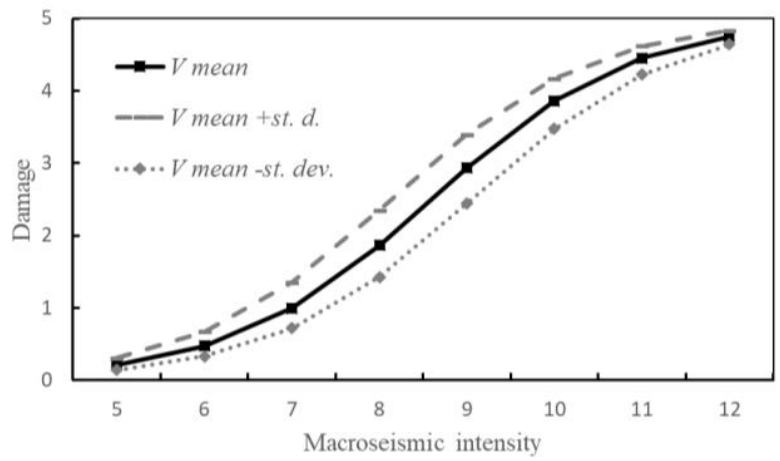

Figure 4. Vulnerability curve of the Scarperia urban stock.

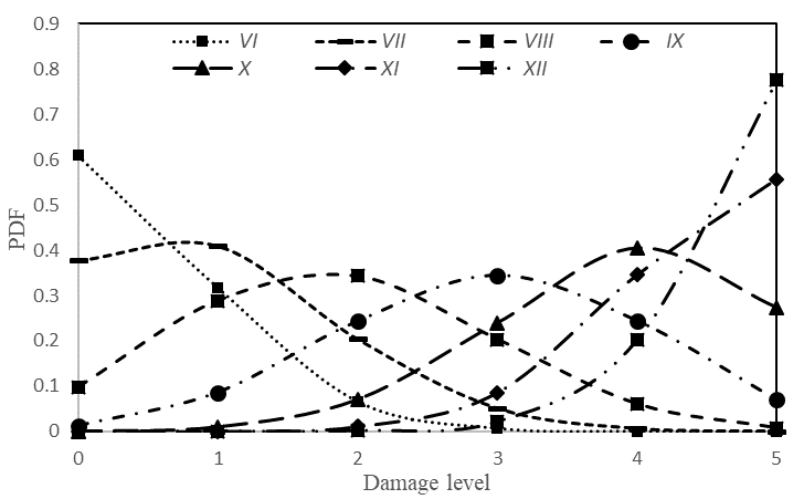

Figure 5. Binomial probability distribution for the centre of Scarperia for different macroseismic intensity levels. 


\subsection{Aggregate Assessment}

An aggregate inside the urban centre has been selected for indepth analyses. Every phase has been developed in a more accurate way. The knowledge-based procedures started at US continue at AL. The selected aggregate presents a rectangular shape and it is located along the main longitudinal street of Scarperia. The buildings are mainly constituted by three storeys and a total of 10 buildings characterizes the system (Figure 6).

In particular, the decrease of scale passing from a GIS environment to a BIM one allowed to gather more specific information that couldn't be caught before. For what concerns the survey, the direct survey conducted has been implement through an indirect Laser Scanner (LS) survey of the façades. The internal measurements have been checked more in detail entering in several buildings and checking the feasibility of the information provided by the archives through the aggregate modelling.

The modelling phases have been developed in the BIM environment Revit (Autodesk) within the following procedure:

- Import of the CAD reconstruction of the different levels;

- Import of the 3-D point cloud;

- 3-D modelling through parametric object at each level;
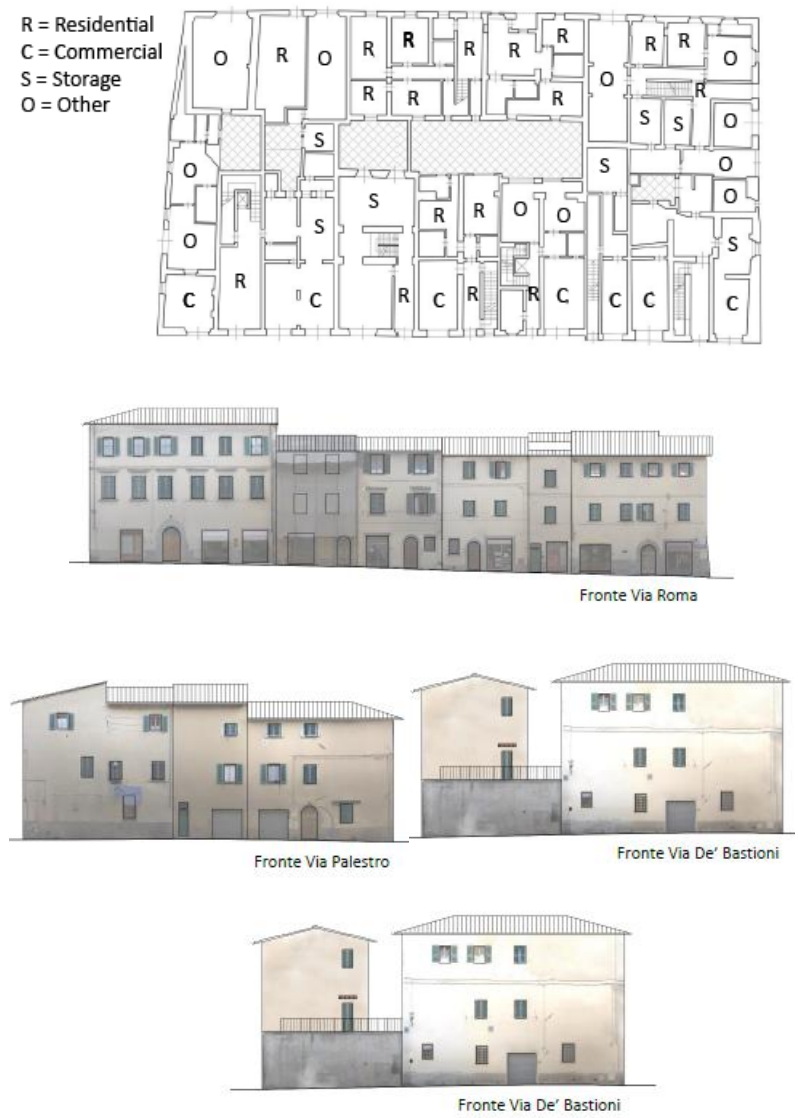

Figure 6. Planimetry of the referred aggregate (ground floor only); photogrammetry of the façades of the buildings (Bonaguidi, 2019).

\subsection{Information modelling and H-BIM management}

Finally, the BIM can be filled of the information provided by the knowledge-based procedures. The LOD, Levels or
Development or Levels or Details (AIA, 2014; NBS, 2016) reached for this type of modelling rounds around a LOD300. In fact, in certain external parts, the quality of the information can even reach higher levels. Regarding to the Levels of Geometry (LOG) the accuracy of the LS survey achieves the LOG500, but, referring to the internal parts, both the LOG than the LOD reach lowest levels.

The $\mathrm{AL}$, as already mentioned, has to manage with the difficulties occurred in the access to the structures and the achievement of complete information. The procedure here presented trys to bypass the problem starting from the documents presented into the municipalities archives, their digitalization and control through 3-D modelling and in-situ inspections. In Figure 7 the quality of the modelling herein proposed is presented. In H-BIM optic, the model becomes itself part of the knowledge-based procedure, since it can be consulted, data exported and uploaded. The masonry and slabs characteristics (when found out) have been modelled. Different documents can be attached to the parametric object presented; in this work the cadastral information taken from the archives, as the thermography campaign done have been linked to the referred elements (Figure 8).

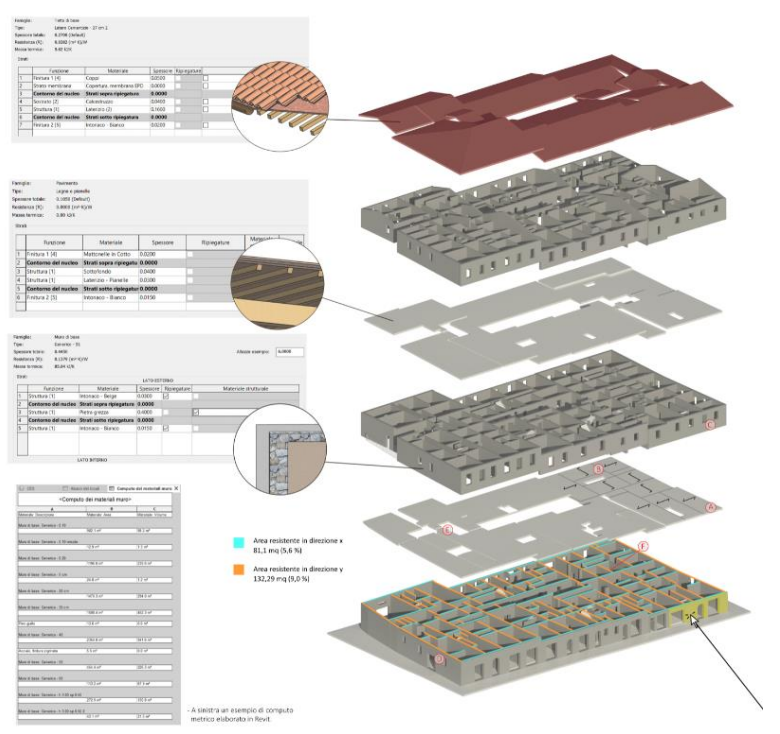

Figure 7. Digital information provided into the H-BIM at AL (Bonaguidi, 2019).

Further developments of the project will allow the possibility to add eventualy destructive-campaigns able to define some mechanical characteristics in order to perform more accurate analytical analysis.

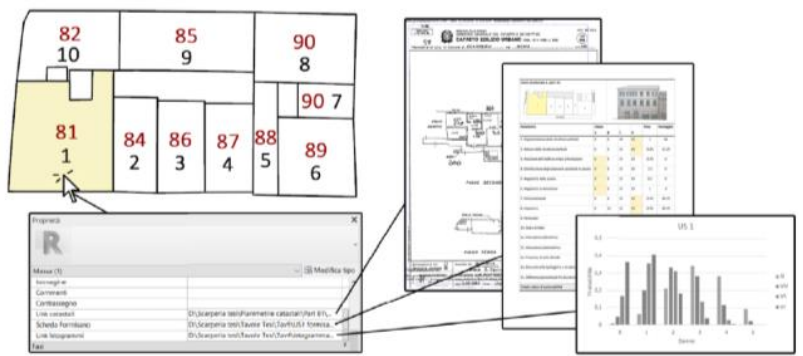

Figure 8. Digital information provided into the H-BIM at AL (Bonaguidi, 2019). 
For what concerns the seismic vulnerability of the urban aggregates, in these phases the BIM becomes in itself both target of the analysis (providing the data in order to adopt the empirical approacheses) and the receptacle of calculations made. For sake of clarity, the application of the empirical methodology will be presented latter in the outcomes of the methodology but we remind how these parts are strictly related and connected to this paragraph.

3.3.1 Analyses of the out-of-plane in the surveys: Laser scanner technology allows the survey of 3-D objects at different scales. Its use at US is testified by several contributions in different countries; the possibilities of a detailed survey are nowadays an effective tool for the management of public spaces from municipalities and governments. Especially in the small urban centers (which Italy is made of) it has already been shown that in case of catastrophic events one of the biggest problems is related to the impossibility to reach every part of the urban areas because of the collapse of the façades over the streets (Dolce et al., 2013).

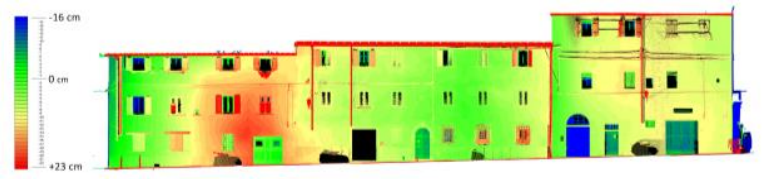

Figure 9. Out-of-plane deformation of a façade of the masonry aggregate.

The use of the point cloud allowed the verification of the out-ofplane deformation of the façades. The non-perfect verticality of the walls in masonry structures is in fact an important alert to point out instability phenomena, overturning mechanisms and irregular loadflows along the masonry walls. In Figure 9 an example with the deformation of a façade is presented. As we can see, the second building from the left present an important deformation at the first level that portends to instability phenomena.

3.3.2 Vulnerability assessment: With the aim of assessing the seismic vulnerability of the urban aggregate, the GNDT second level approach implemented by Formisano et al. (2011) for the urban aggregates has been adopted. Both the methodologies - the original one based on 10 parameters and conceived by Benedetti and Petrini (1984), both the last one with the addition of five new parameters related to the position in the aggregate and the interferences have been used in a relevant way during the last years. Like for the modifiers of the macroseismic scale, the schedule considers the buildings in itself and the buildings inside the aggregate system. The Vulnerability index $I v$ ranges between 0 and 517.5. in order to normalize his value in a macroseismic scale between 0 and 1 , the following expression is adopted:

$V_{i}=\left[\frac{\mathrm{I}_{\mathrm{v}}-\sum_{j=1}^{\mathrm{S}} s_{\min } \times W_{\mathbb{i}}}{\sum_{i=1}^{\mathrm{S}}\left[\left(s_{\max } \times W_{\mathfrak{i}}\right)-\left(s_{\min } \times W_{\mathfrak{i}}\right)\right]}\right]$

Considering the uncertainties that affect some parameters of the methodology proposed, their values have been varied from the lowest to the maximum quantities. In particular, the parameters that have been considered affected by the most important uncertainties are those that involve the role of the wall disposition along the plan and the height of the building, the reduction of resistant areas etc (numbers 4, 5, 6, 10, 15). Parameter n. 10, referring to the quality of the physical condition is still doutful; in fact, only the external façades have been widely assessed. Finally, parameter n. 15 Percentage difference of opening areas among adjacent facades has still been considered uncertain because of the presence of splays and narrowing close to the openings, that have been evaluated just on the ground levels. In Table 2 the results of these variations are showed for the 10 structural units presented in the aggregate objet of this study.

\begin{tabular}{|l|l|l|}
\hline U.S. & $I_{v} \min$ & $I_{v} \max$ \\
\hline 1 & 172.50 & 397.50 \\
2 & 96.25 & 303.75 \\
3 & 53.75 & 283.75 \\
4 & 88.25 & 293.75 \\
5 & 58.75 & 263.75 \\
6 & 103.75 & 333.75 \\
7 & 93.75 & 318.75 \\
8 & 155.00 & 345.00 \\
9 & 161.25 & 328.75 \\
10 & 172.50 & 385.00 \\
mean & 115.58 & 325.38 \\
\hline
\end{tabular}

Table 2 . The vulnerability index for the buildings in aggregate.

Converting the values obtained in macroseismic scale according to (3) two mean values can be obtained, respectively equal to 0.387 and 0.70 . The standard deviation for both cases is equal to 0.06. As we can see, considering the same hypothesis, the dispersions are quite limited. Combining together the two different hypotheses, we obtain a mean value $I_{v}$ equal to 0.54 ; basically, the dispersion increases to 0.22 . This last curve is presented in Figure 10.

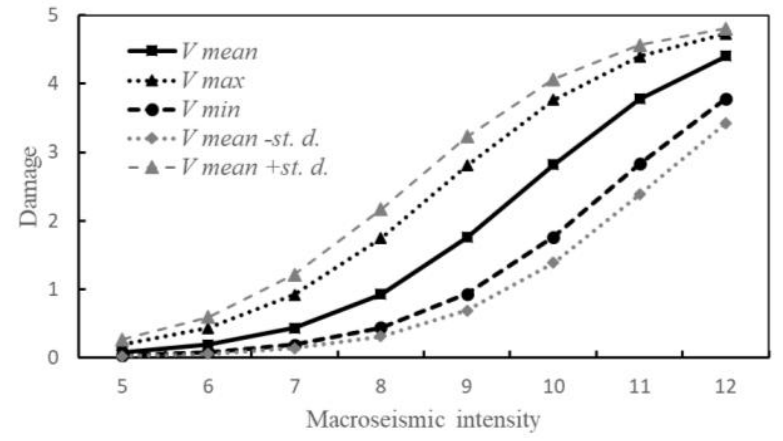

Figure 10. Damage curves for the aggregate studied considering both the preliminary assumptions conducted with the highest dispersion.

3.3.3 Comparison between Damage curves: The damage curves obtained by the 15 parameters GNDT schedule has been compared with the one obtained throught the modiers of Giovinazzi and Lagomarsino.

Figure 11 compares the same aggregate throught the two methodologies. As we can see, the mean value of the macroseismic methodology is close to the one assuming the worst conditions for the internal part of the structural units. This result denounces how, in absence of certain data, the Giovinazzi and Lagomarsino methodology through the proposed modifiers allows to stay more in a most-likely range, paying the price of a low dispersion that could be - compared to the other methodology too much conservative. Otherwise, the Formisano methodology spreads the results highlighting the differences inside the urban stock; it is worth noting like, on the other hand, the second methodology is more affected by the uncertainties presented. For thess reasons, the knowledge-based procedure has to be more accurate as possibile in order to limit the scattering. 


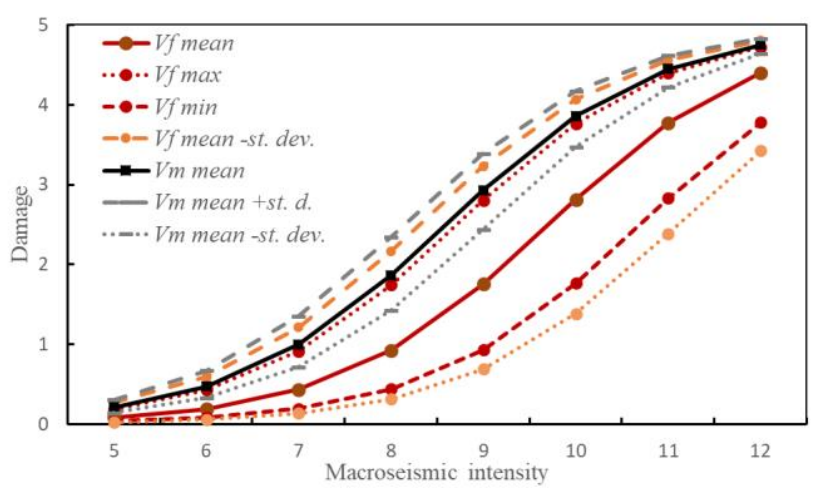

Figure 11. Comparison between the damage curves obtained for the aggregate studied both through the two different methodologies: the Giovinazzi and Lagomarsino macroseismic one (indicated with $m$ ) and the Formisano's one ( $f$ index).

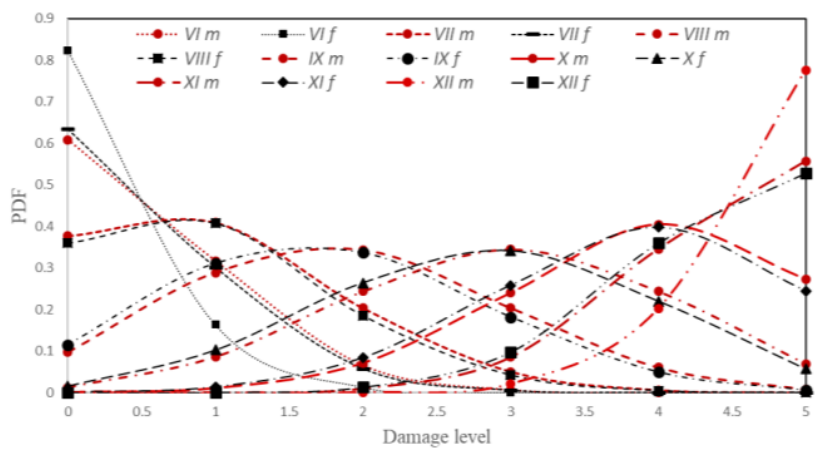

Figure 12. Comparison between the damage probability matrices for the aggregate studied both through the two different methodologies.

Considering the expected damage for Scarperia (grade VI-IX), generally the $f$ index is a level higher than the $m$ index. Indeed, for a IX grade of seismic intensity the most-likely damage considered with Formisano is DL3 while it reaches a DL2 through the Giovinazzi and Lagomarsino method.

\section{CONCLUSIONS}

In this work a multidisciplinary knowledge-based approach for the seismic vulnerability assessment of the historical centre in masonry building aggregates has been presented. The methodology, based on a multiscale approach combining different informatic systems such as GIS and BIM, has been applied to the centre of Scarperia, a municipality located in the Florentine area and characterized by a medium-high seismicity.l

The methodology is based on a multiscale approach and it is aimed at increasing the knowledge path at the aggregate scale through contemporary strategies and tools. To overcome the lack of knowledge related to the impossibility of access to every unit, the proposed approach starts from the cadastral reconstructions obtained by national databases, combined with modern technologies (such as LS surveys) and non-destructive tests. At each scale, the collected data became both the basis of system for the management of the cultural heritage (like historical masonry centre are) and the tool to obtain the information for the seismic analyses.

In this work two different scales have been considered and two empirical methodologies have been applied. At the urban scale, the Giovinazzi and Lagomarsino macroscale approach through the use of modifiers has been adopted, while at the aggregate level the GNDT second level schedule implement by Formisano has been assumed. The results highlight the role of the knowledge path descending the scale of interest. While the macroscale approach keeps a less sensitivity to the knowledge obtained (so that the level of information put into the GIS is even probably "too much" for the simplicity of the methodology in itself), the Formisano schedule denounces a higher sensitivity. In conclusion, Giovinazzi and Lagomarsino modifiers are more conservative and they provide, for each intensity, a damage state generally one level higer than Formisano's procedure. Mechanical analyses are now expected in order to clarify the relationships between the two methodologies, in order to fix out some staple for the calibration of the empirical methods.

For what concern the city centre of Scarperia, the results evicenced its vulnerability and the necessity of adopting strengthening solutions. The work points out how the informative systems provided during the methodology herein presented and used for the analyses can still be at the basis for further phases of design of interventions or retrofitting. Finally, their potential can be expressed through the updating of data for a management of the public areas.

\section{ACKNOWLEDGEMENTS}

This research belongs to the Protocol signed between the Municipality of San Piero e Scarperia and Department of Architecture of the University of Florence for the seismic vulnerability assessment of the historical centre of Scarperia.

\section{REFERENCES}

AIA, 2015. The American Institute of Architects', BIM forum, Level of Development (LOD) specification, http://www.aiacc.org/2015/11/

Benedetti, D., Petrini, V., 1984. Sulla vulnerabilità si sismica di edifici in muratura: Un metodo di valutazione, L'Industria Delle Costruzioni (in Italian)

Bento, R., 2019. An Interdisciplinary Approach to the Seismic Assessment of Built Cultural Heritage: Case Studies in Lisbon and Outskirts, R. Aguilar et al. (Eds.): Structural Analysis of Historical Constructions, RILEM Bookseries 18, pp. 3-18, 2018. https://doi.org/10.1007/978-3-319-99441-3_1

Bonaguidi, L., 2019. Vulnerabilità sismica: metodo sperimentale per lo studio di un aggregato strutturale a Scarperia, Bachelor's degree in Architecture, University of Florence. (in Italian)

Catulo, R., Falcão, A. P., Bento, R., Ildefonso, S., 2018. Simplified evaluation of seismic vulnerability of Lisbon Heritage City Centre based on a 3DGIS-based methodology. Journal of Cultural Heritage 32: S1296207417301073, 2018

Cavaleri, L., Di Trapani, F., Ferrotto, M.F., 2017. A new hybrid procedure for the definition of seismic vulnerability in Mediterranean cross-border urban areas, Nat. Hazards, vol. 86, 517-541. http://dx.doi.org/10.1007/s11069-016-2646-9

Colucci, E., Noardo, F., Matrone, F., Spanò, A., Lingua, A., 2018. High-Level-of-Detail semantic 3D representation of Architectural Heritage, Int. Arch. Photogramm. Remote Sens. Spatial Inf. Sci., XLII-4, 107-114, https://doi.org/10.5194/isprsarchives-XLII-4-107-2018 
D’Altri, A. M., Sarhosis, V., Milani, G., Rots, J., Cattari, S., Lagomarsino, S., Sacco, E., Tralli, A., Castellazzi, G., De Miranda, S., 2019. A review of numerical models for masonry structures, Numerical Modeling of Masonry and Historical Structures, From Theory to Application, Woodhead Publishing Series in Civil and Structural Engineering, 3-53.

Dolce, M., Speranza, E., Di Pasquale, G., Giordano, F., Bocchi, F.; 2013. Indici di operatività per la valutazione della Condizione Limite di Emergenza (CLE). $33^{\circ}$ Convegno Nazionale GNGTS, Trieste (in Italian)

EC 8-3, 2005. Design of structures for earthquake resistance, part 3: strengthening and repair of buildings. European standard EN 1998-3. European Committee for Standardization (CEN), Brussels.

Federal Emergency Management Agency, FEMA-356, 2000. Prestandard and Commentary for Seismic Rehabilitation of Buildings, Washington DC.

Formisano, A., Florio, G., Landolfo, R., Mazzolani, F. M., 2011. Un metodo per la valutazione su larga scala della vulnerabilità sismica degli aggregati storici. XV Convegno ANDIS - L'Ingegneria Sismica in Italia.

Galassi, S., Ruggieri, N. Tempesta, G., 2018. A Novel Numerical Tool for Seismic Vulnerability Analysis of Ruins in Archaeological Sites, International Journal of Architectural Heritage, doi: 10.1080/15583058.2018.1492647

Giovinazzi, S., Lagomarsino, L., 2004. A macroseismic model for the vulnerability assessment of buildings, 13th World Conference on Earthquake Engineering, Vancouver, Canada.

Greco, A., Lombardo, G., Pantò, B., Famà, A., 2018. Seismic Vulnerability of Historical Masonry Aggregate Buildings in Oriental Sicily. International Journal of Architectural Heritage, 1-24. doi:10.1080/15583058.2018.1553075

Grunthal, G., 1998. European Macroseismic Scale. Chaiers du Centre Européen de Géodynamique et de Séismologie, vol. 15 Luxembourg.

Gruppo Nazionale per la Difesa dai Terremoti - GNDT-SSN, 1994. Scheda di esposizione e vulnerabilità e di rilevamento danni di primo e secondo livello (muratura e c.a.). (in Italian).

Lagomarsino, S., 2006. On the vulnerability assessment of monumental buildings. Bulletin of Earthquake Engineering, 4(4), 445-463. doi:10.1007/s10518-006-9025-y

Lantada, N., Irizarry, J., Barbat, A. H., Goula, X., Roca, A., Susagna, T., Pujades, L. G., 2010. Seismic hazard and risk scenarios for Barcelona, Spain, using the Risk-UE vulnerability index method, Bullettin of Earthquake Engineering 8(2):201229. doi: 10.1007/s10518-009-9148-Z

Maio, R., Vicente, R., Formisano, A., Varum, H., 2015. Seismic vulnerability of building aggregates through hybrid and indirect assessment techniques. Bulletin of Earthquake Engineering. https://doi.org/10.1007/s10518-015-9747-9

Martínez-Cuevas, S., Benito, M. B., Cervera, J., Morillo, M. C., Luna, M., 2017. Urban modifiers of seismic vulnerability aimed at Urban Zoning Regulations. Bulletin of Earthquake Engineering. https://doi.org/10.1007/s10518-017-0162-2
Matrone, F., Colucci, E., De Ruvo, V., Lingua, A., Spanò, A., 2019- HBIM in a semantic 3D GIS Database, Int. Arch. Photogramm. Remote Sens. Spatial Inf. Sci., XLII-2/W11, 857865, https://doi.org/10.5194/isprs-archives-XLII-2-W11-8572019.

NBS, 2016. National BIM report. https://www.thenbs.com. Accessed date 29.03.2018

Ruggieri, N., Galassi, S., Tempesta, G., 2018. Pompeii's Stabian Baths. Mechanical behaviour assessment of selected masonry structures during the 1 st century seismic events, International Journal of Architectural Heritage, 12(5), 859-878. doi: 10.1080/15583058.2017.1422571.

Senaldi, I., Magenes, G., Penna, A., 2010. Numerical Investigations on the Seismic Response of Masonry Building Aggregates. Advanced Materials Research. https://doi.org/10.4028/www.scientific.net/amr.133-134.715

Senaldi, I. E., Guerrini, G., Comini, P., Graziotti F., Penna, A., Beyer, K., Magenes, G., 2019. Experimental seismic performance of a half scale stone masonry building aggregate, Bullettin of Earthquake Engineering, 18, 609-643. https://doi.org/10.1007/s10518-019-00631-2

Talon, A., Cauvin, C., Chateauneuf, A. 2017. State of the Art of HBIM to Develop the HBIM of the HeritageCare Project. International Journal of 3-D Information Modeling, 6(3), 3343. doi:10.4018/ij3dim.2017070103

Valente, M., Milani, G., Grande, E., Formisano, A., 2019. Historical masonry building aggregates: advanced numerical insight for an effective seismic assessment on two row housing compounds. Engineering Structures, 190, 360-379. doi:10.1016/j.engstruct.2019.04.025

Vicente, R., Parodi, S., Lagomarsino, S., Varum, H., Mendes Silva. J. A. R., 2011: Seismic vulnerability and risk assessment: case study of the historic city centre of Coimbra, Portugal, Bullettin of Earthquake Engineering 9:1067-1096 doi:10.1007/s10518-010-9233-3. 